\title{
Metallomesogens as stationary phases for ligand exchange gas chromatography - Part I. The use of nickel and zinc complexes of 4-decanoxydithiobenzoic acid for the separation of polycyclic aromatic hydrocarbons and dialkyl sulfides
}

\author{
Cho-Chun Hu, Chuen-Ying Liu* \\ Department of Chemistry, National Taiwan University, Taipei, Taiwan
}

Received 7 January 1996; accepted 29 April 1996

\begin{abstract}
Metallomesogens based on 4-decanoxydithiobenzoic acid (DDTBA) metal complexes were prepared and applied to the separation of polycyclic aromatic hydrocarbons (PAHs) and dialkyl sulfides. The mesophase of DDTBA-Ni was stable at temperatures ranging from $140^{\circ}$ to $230^{\circ} \mathrm{C}$ and that of DDTBA-Zn was stable at temperatures ranging from $137^{\circ}$ to $173^{\circ} \mathrm{C} .5 \%$ of each material was coated on Chromosorb and then packed in a $2.1 \mathrm{~m} \times 3.2 \mathrm{~mm}$ i.d. spiral glass column. Factors affecting the retention time and the selectivity were studied. The results indicate that the retention time is greatly affected by the molecular geometry in addition to the vapor pressure and polarity of the solutes, and that a DDTBA-Ni column is adequate for the separation of PAHs, whereas a DDTBA-Zn column is suitable for the separation of dialkyl sulfides. Van't Hoff plots as a function of temperature indicate that phase transitions were occurring. Thermodynamic properties of the analytes in this system were also studied.
\end{abstract}

Keywords: Gas chromatography; Metallomesogens; Ligand exchange GC; Polycyclic aromatic hydrocarbons; Dialkyl sulfides

\section{Introduction}

Liquid crystals have been used as stationary phases in gas chromatography for over 30 years [1]. Among various organic substances of a liquid crystal nature, more than 200 mesogenic stationary phases of nematic, smectic and cholesteric types are used in chromatography at present [2]. Since 1977, Giroud and Mueller-Westerhoff [3] reported some meso-

\footnotetext{
${ }^{*}$ Corresponding author. Fax: 88623636359.
}

morphic dithiolene complexes of $\mathrm{Ni}(\mathrm{II})$ and this work is widely accepted as being the beginning of systematic research into metal-containing systems. Currently, there is much interest in the synthesis and characterization of liquid crystalline material containing metal atoms, because of the potential to modify many physical properties via the inclusion of a metal center [4].

Dithiocarbamates and their derivatives are important analytical reagents. In a previous paper [5] we reported a ligand-exchange gas chromatography (LEGC) separation of dialkyl sulfides, using dithio- 
carbamate resin-metal complexes as the stationary phase. Recently, the mesomorphic behavior [3] exhibited by dithiolene metal complexes has led us to investigate the application of metallomesogens as stationary phases in LEGC. However, there are only a few reports relating the usage of metallomesogens as stationary phases in chromatography [6-8]. The present work involved a preparation of metallomesogens based on the p-decanoxydithiobenzoic acidmetal complexes and applying them to the separation of dialkyl sulfides and polycyclic aromatic hydrocarbons (PAHs).

\section{Experimental}

\subsection{Apparatus}

The experiments were carried out with a Shimadzu (Kyoto, Japan) Model GC-9A gas chromatograph equipped with a flame ionization detector. The carrier gas was high purity grade nitrogen. The phase behavior was determined using a polarizing optical microscopy (BH-2, BHT, Olympus, Japan) equipped with a temperature controller (TMS 92, Linkam, UK) and a differential scanning calorimetry (DSC)(Model 910, TA, USA) at a scan rate of $10^{\circ} \mathrm{C} \mathrm{min}-1$.

\subsection{Reagents}

Most chemicals used were of analytical reagent grade from Merck (Darmstadt, Germany). All liquid reagents and solvents used in moisture-sensitive reactions were distilled and collected over type $4 \AA$ molecular sieves. All solid materials used in moisture-sensitive reactions were dried at $110^{\circ} \mathrm{C}$ for $24 \mathrm{~h}$ prior to the experiment.

\subsection{Synthesis of p-decaoxybromobenzene}

The mixture of 4-bromophenol (9.51 g), potassium hydroxide $(3.10 \mathrm{~g})$ and potassium iodide $(0.2 \mathrm{~g})$ in ethanol were refluxed for $1 \mathrm{~h}$. Next 1-bromodecane $(11.06 \mathrm{~g})$ was added dropwise with stirring and refluxed for $16 \mathrm{~h}$. Then $30 \%$ potassium hydroxide aqueous solution was added and the mixture was refluxed for an additional $2 \mathrm{~h}$. After the reaction mixture had been cooled and concentrated under reduced pressure, the residue was extracted with dichloromethane. The organic layer was washed with pure water and dried with anhydrous magnesium sulfate. The mixture was filtered and concentrated under reduced pressure. The purity was tested by thin-layer chromatography and the structure of the compound was confirmed by melting point, elemental analysis and IR spectrometry.

\subsection{Synthesis of p-decaoxydithiobenzoic acid from p-decaoxybromobenzene}

The synthesis of this compound was carried out according to the [4] with slight modification. For preparation of the Grignard reagent, the reaction was conducted in the minimum amount of THF.

\subsubsection{Grignard reaction}

Magnesium turnings $(0.48 \mathrm{~g})$ were placed in a twoneck round-bottom flask $(100 \mathrm{ml})$ equipped with an additional funncl, a reflux condenser and a mechanical stirrer. A few drops of $p$-decaoxybromobenzene and a small crystal of iodine were introduced to initiate the reaction. Then $p$-decaoxybromobenzene $(6.3 \mathrm{~g})$ in dry tetrahydrofuran $(20 \mathrm{ml})$ was added dropwise to the reaction mixture which was under a nitrogen atmosphere. They were heated to reflux and heating was continued for an additional $2 \mathrm{~h}$. The round-bottom flask was sealed with a rubber septum.

\subsubsection{Synthesis of p-decaoxydithiobenzoic acid (DDTBA)}

Carbon disulfide $(2.5 \mathrm{ml})$ was introduced dropwise into a round-bottom flask which contained the above Grignard reagent in an ice bath. The mixture turned red immediately and was stirred for an additional $2 \mathrm{~h}$. Next, ice $(200 \mathrm{~g})$ and concentrated hydrochloric acid $(100 \mathrm{ml})$ were added to the reaction mixture, which was extracted with diethyl ether, then dried with magnesium sulfate. The ether was removed with a rotary evaporator and the product was precipitated at $-15^{\circ} \mathrm{C}$. Because of the relatively low stability of the free acid, the compound was stored in the form of its sodium salt or directly reacted with metal salts in a mix-solvent of methanol and dichloromethane $50 \%$, $\mathrm{v} / \mathrm{v})$. 


\subsection{Preparation of metal complexes}

The preparation of metal complexes was achieved by dropwise addition of a methanol solution of nickel chloride hexahydrate or zinc acetate dihydrate in an inert atmosphere to a dichloromethane-methanol $(50 \%, v / v)$ mixture containing an excess of the sodium salt of $p$-decaoxydithiobenzoic acid (DDTBA) (2.1:1 molar ratio). After the solution had been stirred for $8 \mathrm{~h}$, the resulting nickel complex precipitate was collected by filtration, washed sequentially with pure water, methanol and diethyl ether, then recrystallized from hot toluene to give the product as deep blue crystals. The zinc complex, which had a reddish orange color, was obtained similarly after careful crystallization from toluene/ hexane.

The metallomesogens $(5 \%, \mathrm{w} / \mathrm{w})$ were deposited on Chromosorb W AW-DMCS (80-100 mesh) and conditioned for $1 \mathrm{~h}$ at $60^{\circ} \mathrm{C}$ from a toluene solution. Glass columns $(2.1 \mathrm{~m} \times 3.2 \mathrm{~mm}$ i.d. $)$ were filled with the support and then conditioned for $8 \mathrm{~h}$ at $150^{\circ} \mathrm{C}$.

\subsection{Measurement of dissolved ammonia in aqueous solution}

For the determination of coordination unsaturation of the central metal ion in the metallomesogens, ammonium chloride solution $(0.04 \mathrm{M}, 25 \mathrm{ml})$ was added to each metallomesogen $(0.2 \mathrm{~g})$ in a polyethylene bottle $(100 \mathrm{ml})$. The mixture was stirred. After $24 \mathrm{~h}$, the residue was filtered and the ammonia in the filtrate was determined electrochemically via an ammonia electrode (model 95-12, Orion, USA) attached to a direct concentration readout spccific ion meter (Orion Model EA 940).

\section{Results and discussion}

\subsection{Characterization of the prepared stationary phases}

Differential scanning calorimetry (DSC) measurements are the simplest means for detecting phase transitions in liquid crystals. Temperatures of the phase change (Table 1) agree with the literature
Table 1

Phase transitions of metallomesogens

\begin{tabular}{ll}
\hline Compounds & Phase transitions $\left({ }^{\circ} \mathrm{C}\right)^{\mathrm{a}}$ \\
\hline DDTBA-Ni & K $140.2-$ SmH 167.5 - SmC 235 - I \\
DDTBA-Zn & K $131.4-$ SmC $160.9-$ N 173.2 - I \\
\hline
\end{tabular}

${ }^{\mathrm{a}} \mathrm{K}=$ solid crystalline, $\mathrm{Sm}=$ smectic, $\mathrm{N}=$ nematic, I=isotropic.

values [4]. A comparison of the DSC measurements for bare Chromosorb, DDTBA-Zn and DDTBA-Ni coated on Chromosorb was made. It shows that both the metal complexes coated on Chromosorb retained their liquid crystal property. Owing to the weaker intermolecular hydrogen bonds, dithiobenzoic acid itself does not show any mesomorphic behavior, unlike the isostructural 4-alkoxybenzoic acid [4]. Since the dithiobenzoic acid and Chromosorb itself do not show any mesomorphic behavior, the liquid crystal property can be attributed to the metal complex coated on the Chromosorb.

Preliminary tests show that the nickel complex which exhibits the higher transition temperature is adequate for the separation of PAHs, while the zinc complex which exhibits a lower transition temperature is more suitable for the separation of dialkyl sulfides. In this investigation, the boiling points of most PAHs are in the range of $200^{\circ}$ to $300^{\circ} \mathrm{C}$. Hence the amount of coating on the Chromosorb is around $5 \%$ [9].

Since the principle of LEGC is a process in which complex-forming compounds are separated through the formation and breaking of labile coordinate bonds to a central metal atom, an ammonium ion selective electrode is applied for the first time to metallomesogens in order to ascertain if their properties are suitable as stationary phases of LEGC. The results show that the ammonia consumed by DDTBA-Ni is $0.98 \mathrm{mmol} / \mathrm{mmol}$ and that for DDTBA-Zn is $1.09 \mathrm{mmol} / \mathrm{mmol}$. In other words, the coordination environment around the metal is appropriate for this purpose.

\subsection{Separation of PAHs and dialkyl sulfides}

The optimal conditions for the separation of dialkyl sulfides and PAHs obtained by a univariate procedure, are provided in Table 2 . 
Table 2

Optimum conditions utilized for the separation of dialkyl sulfides and polyaromatic hydrocarbons with a packed column

Stationary phase $5 \%$ DDTBA-Zn ${ }^{a}$, DDTBA-Ni ${ }^{b}$ on Chromosorb

Dimensions of the column Injector temperature Column temperature Carrier gas

Flow rate of carrier gas Total weight of Chromosorb Total weight of liquid crystals Molecular weight of liquid crystal

${ }^{a}$ For dialkyl sulfides. ${ }^{b}$ For polyaromatic hydrocarbons. ${ }^{c} 50^{\circ} \mathrm{C}(3$ min) to $100^{\circ} \mathrm{C}$ at $5^{\circ} \mathrm{C} \mathrm{min}^{-1}$. d $50^{\circ} \mathrm{C}(7 \mathrm{~min})$ to $135^{\circ} \mathrm{C}$ at $5^{\circ} \mathrm{C}$ $\min ^{-1}$.

\subsubsection{Polycyclic aromatic hydrocarbons}

The separation of PAHs on DDTBA-Ni is shown in Fig. 1. The results show that the retention time of 2-methylnaphthalene is nearly the same as that of 1methylnaphthalene and that it is difficult to separate them in the conditions mentioned. The boiling point of 1-methylnaphthalene is $3^{\circ}$ higher than that of 2methylnaphthalene, but the length to breadth ratio of the latter molecule (1.381) is larger than the former (1.208). Clearly in the smectic region of DDTBA-Ni, the solute geometrical factor (length to breadth ratio) is a significant factor, in addition to the boiling points of the solutes. The same phenomenon was also observed in Lee's work on the separation of 2-/1methylphenanthrene [10]. The mechanism for separation may be dependent on other factors as well. Moreover, the $\mathrm{C}=\mathrm{C}$ in the PAHs might also form a chemical bond with the empty orbital of the central metal ion of the metallomesogens on the Chromosorb. The more the ring is fused, the stronger is the chemical bond formation with the stationary phase and the greater is the retention time.

Fig. 2A and B show the dependence of $\log k^{\prime}$ vs. reciprocal temperature for the PAHs. The adsorbent phase transition for DDTBA-Ni (Fig. 2A) is more clearly seen in comparison with that for DDTBA-Zn (Fig. 2B). Since sorbents with liquid crystals tend to improve the separation, the behavior provides evidence of better separation of PAHs with DDTBA$\mathrm{Ni}$ than with DDTBA-Zn.

The infinite-dilution solute partial molar enthalpy and entropy of solution can be obtained by means of

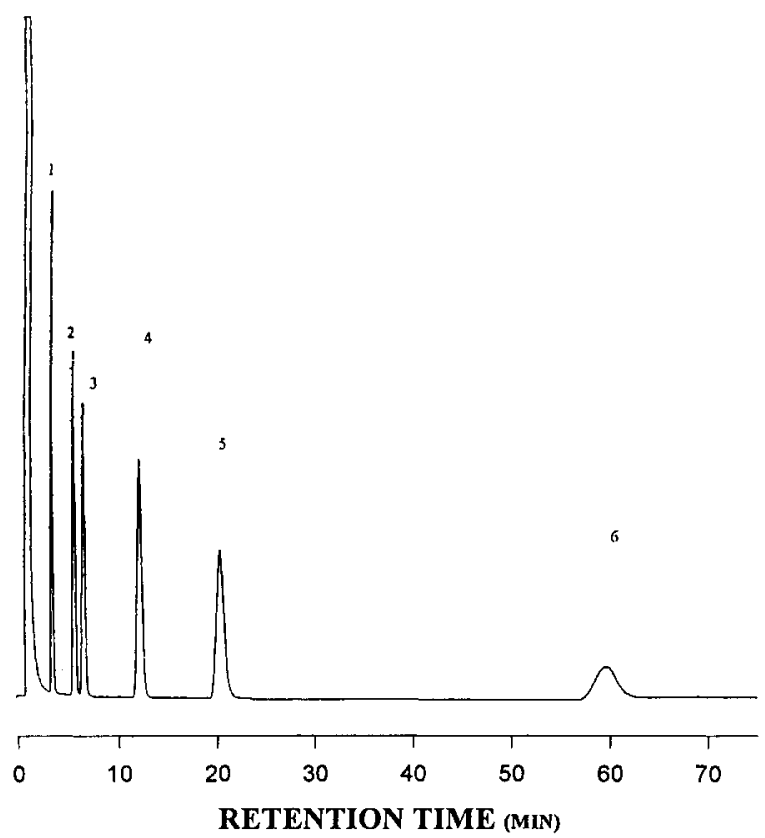

Fig. 1. Chromatogram for the separation of polycyclic aromatic hydrocarbons. Stationary phase: 5\% DDTBA-Ni coated on Chromosorb, $2.1 \mathrm{~m} \times 3.2 \mathrm{~mm}$ i.d. glass column; Oven temp.: $165^{\circ} \mathrm{C}$; Injector temp.: $250^{\circ} \mathrm{C}$; Mobile phase: nitrogen gas, flow rate: $50 \mathrm{ml} \mathrm{min}^{-1}$; Sample volume: $1 \mu \mathrm{l}$. Peak identification: (1) naphthalene $\left(t_{1}=3.58 \mathrm{~min}\right)$; (2) 1-methylnaphthalene and 2-methylnaphthalene $\left(t_{\mathrm{r}}=5.81 \mathrm{~min}\right)$; (3) biphenyl $\left(t_{\mathrm{r}}=6.76 \mathrm{~min}\right)$; (4) acenaphthene $\left(t_{\mathrm{r}}=12.43 \mathrm{~min}\right) ; \quad(5)$ fluorene $\quad\left(t_{\mathrm{r}}=20.85 \mathrm{~min}\right) ; \quad(6)$ anthracene $\left(t_{\mathrm{r}}=60.90 \mathrm{~min}\right)$.

the following equations $[11-13]$ :

$\ln V_{\mathrm{g}}^{\mathrm{o}}=\frac{-\Delta \mathrm{H}}{\mathrm{RT}}+\frac{\Delta \mathrm{S}}{\mathrm{R}}-\ln \left(\frac{M}{273.3 R}\right)$,
$V_{\mathrm{g}}^{\mathrm{o}}=V^{\prime} / W$
$V^{\prime}=\left(t_{\mathrm{r}}-t_{\mathrm{o}}\right) \times f$

where $t_{\mathrm{r}}$ is the retention time of the sample; $t_{\mathrm{o}}$ is the retention time of the nonadsorbed substance; $V^{\prime}$ is the retention volume; $f$ is the flow rate of the carrier gas; $W$ is the weight of the stationary phase; $M$ is the molecular weight of the stationary phase; and $V_{\mathrm{g}}^{\mathrm{o}}$ is the specific retention volume.

Plotting $\ln V_{\mathrm{g}}^{\mathrm{o}}$ vs. $1 / T$ as shown in Fig. $3 \mathrm{~A}$ and $\mathrm{B}$ yields $\Delta H$ (from the slope) and $\Delta S$ (from the intercept). The results are summarized in Table 3 . As the carbon number increases, the attractive force for the PAHs on the metallomesogens increases. This 

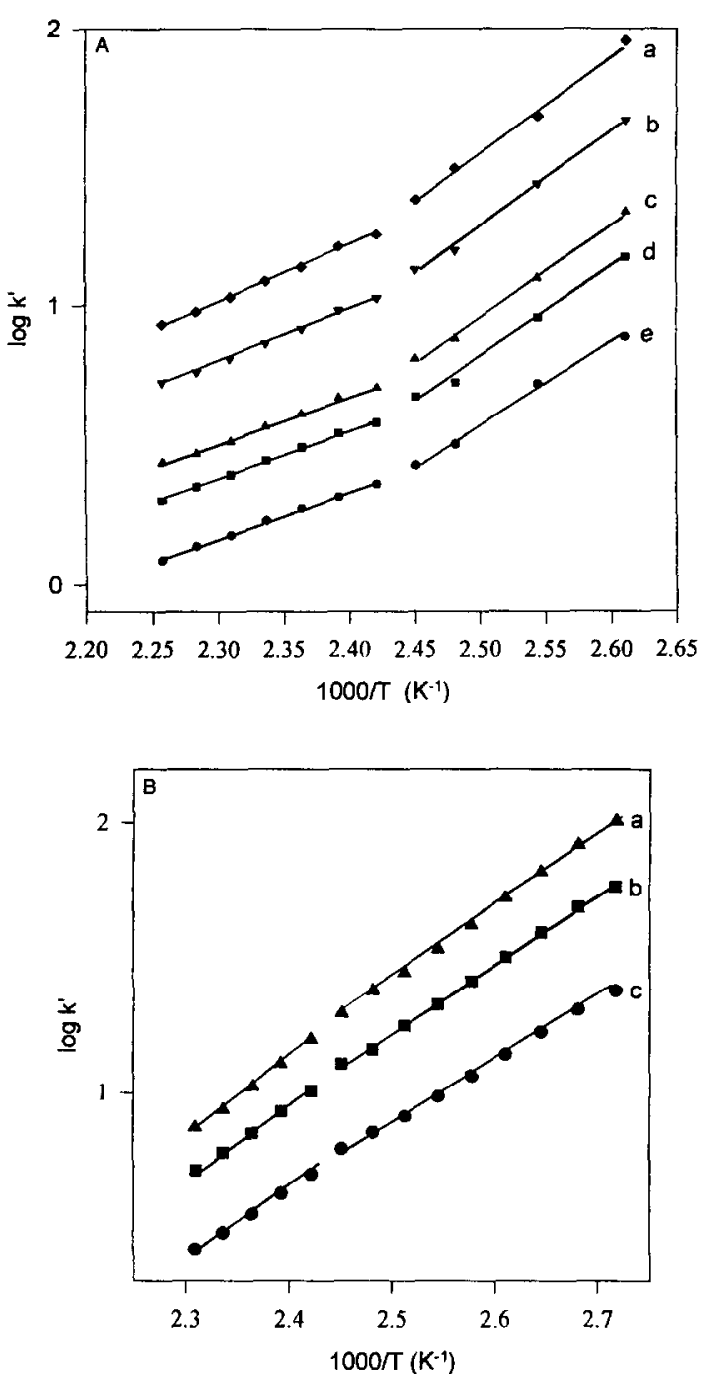

Fig. 2. Van't Hoff plot of polycyclic aromatic hydrocarbons. (A) Stationary phase: $5 \%$ DDTBA-Ni coated on Chromosorb; $2.1 \mathrm{~m} \times 3.2 \mathrm{~mm}$ i.d. glass column; Mobile phase: nitrogen gas, flow rate: $50 \mathrm{ml} \mathrm{min}^{-1}$. Sample: (a) naphthalene; (b) 1-methylnaphthalene; (c) biphenyl; (d) acenaphthene; (e) fluorene. (B) Stationary phase: 5\% DDTBA-Zn coated on Chromosorb; $2.1 \mathrm{~m} \times 3.2 \mathrm{~mm}$ i.d. glass column; Mobile phase: nitrogen gas, flow rate: $50 \mathrm{ml} \mathrm{min}^{-1}$. Sample: (a) isopropylbenzene; (b) naphthalenc; (c) 1-methylnaphthalene.

phenomenon might be attributed to $\pi-\pi$ interactions or van der Waals forces between the analyte and the stationary phase. There is no significant variation in the $\Delta S$ value when the PAHs are dissolved in the metallomesogens, except for 1-methylnaphthalene, 2-
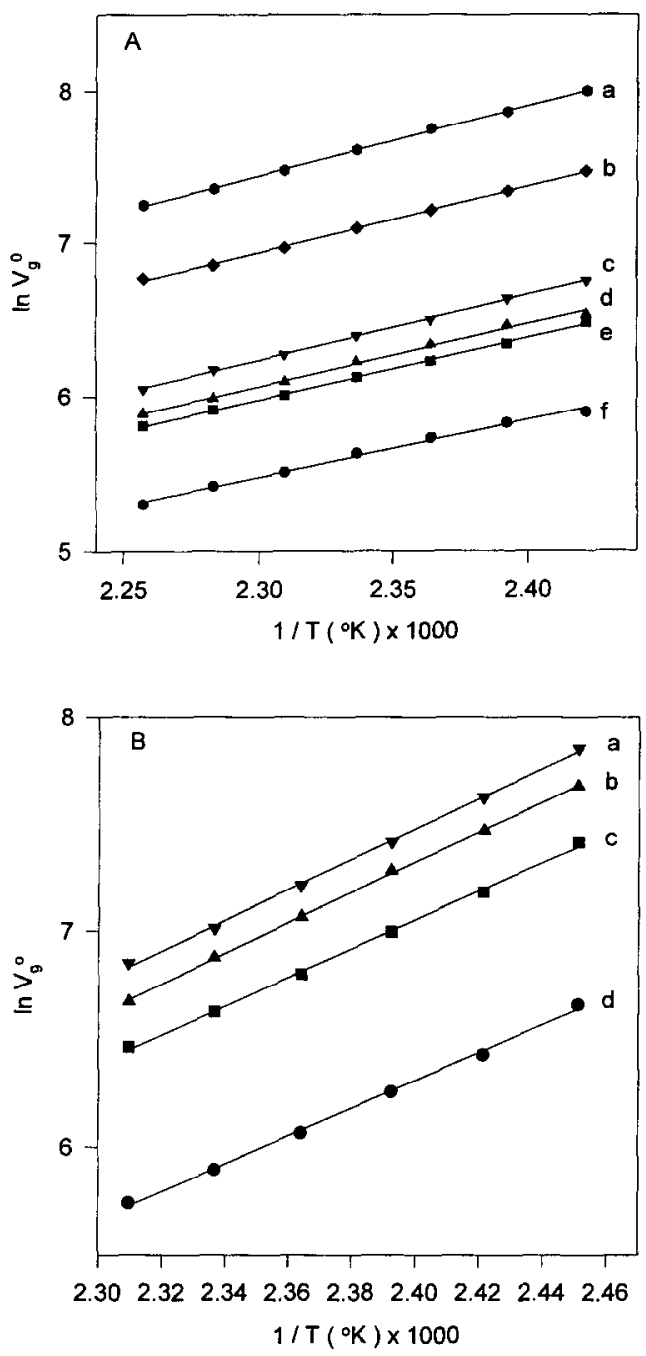

Fig. 3. Temperature dependence of specific retention volume for polyaromatic hydrocarbons. (A) Conditions as Fig. 2A. Sample: (a) fluorenc; (b) acenaphthene; (c) biphenyl; (d) 1-methylnaaphthalene; (e) 2-methylnaphthalene; (f) naphthalene. (B) Conditions as Fig. 2B. Sample: (a) 1-methylnaphthalene; (b) 2-methylnaphthalene; (c) naphthalene; (d) isopropylbenzene.

methylnaphthalene and biphenyl. The result is rational since most PAHs have a rigid structure, while biphenyl,1-methylnaphthalene and 2-methylnaphthalene could rotate more freely than the others. By comparing the $\Delta \mathrm{H}$ and $\Delta \mathrm{S}$ values for PAHs on both stationary phases (Table 3 ), it is found that $\Delta H$ and $\Delta \mathrm{S}$ are larger between PAHs and DDTBA-Zn than between PAHs and DDTBA-Ni. The structure of 
Table 3

Thermodynamic parameters of analytes dissolution in the liquid crystal phases of DDTBA-Ni and DDTBA-Zn

\begin{tabular}{llll}
\hline $\begin{array}{l}\text { Stationary } \\
\text { phase }\end{array}$ & Analyte & $\begin{array}{l}-\Delta \mathrm{H} \\
(\mathrm{kJ} \mathrm{mol}\end{array}$ & $-\Delta \mathrm{S}$ \\
\hline DDTBA-Ni & \multicolumn{1}{c}{$\begin{array}{l}\text { Polyaromatic hydrocarbons } \\
\text { naphthalene }\end{array}$} & 1.75 & 3.08 \\
& 2-methylnaphthalene & 1.94 & 3.29 \\
& 1-methylnaphthalene & 1.96 & 3.28 \\
& biphenyl & 2.02 & 3.36 \\
& acenaphthene & 2.05 & 3.08 \\
& phenanthrene & 2.19 & 3.17 \\
& Dialkyl sulfides & & \\
& di-t-butyl sulfide & 2.92 & 5.16 \\
& di- $n$-butyl sulfide & 3.09 & 5.19 \\
& di- $n$-hexyl sulfide & 3.30 & 5.62 \\
& & & \\
Polyaromatic hydrocarbons & \\
DDTBA-Zn & isopropylbenzene & 2.16 & 4.09 \\
& naphthalene & 2.21 & 3.99 \\
& 2-methylnaphthalene & 2.40 & 4.32 \\
& 1-methylnaphthalene & 2.42 & 4.30 \\
& Dialkyl sulfides & & \\
& di- $t$-butyl sulfide & 3.13 & 5.69 \\
di- $n$-butyl sulfide & 3.29 & 5.71 \\
& di- $n$-hexyl sulfide & 3.48 & 6.07 \\
\hline
\end{tabular}

DDTBA- $\mathrm{Zn}$ is more flexible than that of DDTBA$\mathrm{Ni}$. This might be the result of a stronger interaction between the analyte and DDTBA-Zn.

\subsubsection{Dialkyl sulfides}

Fig. 4 shows an example of the separation of dialkyl sulfides on Chromosorb coated with DDTBA$\mathrm{Zn}$. Because of the high volatility and the wide boiling point range of the investigated sulfur compounds, the less volatile compounds could not be separated efficiently at low temperature. So temperature programming was carried out in two steps. Fig. 5 shows the dependence of $\log \mathrm{k}^{\prime}$ vs. reciprocal temperature for dialkyl sulfides. A phase transition is observed with some difficulty. Takayanagi et al. [7] first described the partition of dialkyl sulfides into copper stearate which behaves as a liquid crystal in the temperature range $115-120^{\circ} \mathrm{C}$, in addition to a ligand exchange reaction, although no quantitative data was given. The same mechanism might be involved in our system. The lack of clarity of the phase transition is probably due to adsorption on the stationary phase surface and the thin coating of

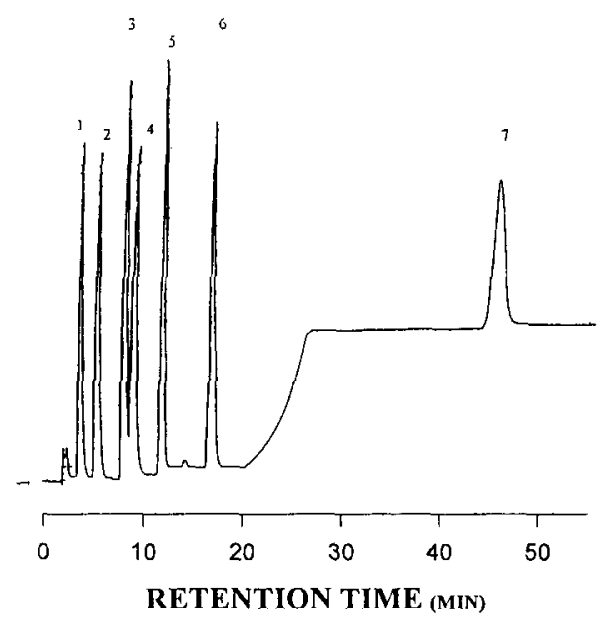

Fig. 4. Chromatogram for the separation of dialkyl sulfides. Stationary phase: $5 \%$ DDTBA-7n coated on Chromosorb, $2.1 \mathrm{~m} \times 3.2 \mathrm{~mm}$ i.d. glass column; Oven temp.: $50^{\circ} \mathrm{C}(3 \mathrm{~min})$ to $100^{\circ} \mathrm{C}(7 \mathrm{~min})$ at $5^{\circ} \mathrm{C} \mathrm{min}^{-1}$ to $135^{\circ} \mathrm{C}(20 \mathrm{~min})$ at $5^{\circ} \mathrm{Cmin}^{-1}$; Injection temp.: $180^{\circ} \mathrm{C}$; Mobile phase: nitrogen gas, flow rate: $40 \mathrm{ml} \mathrm{min}^{-1}$. Peak identification: (1) diethyl sulfide $\left(t_{\mathrm{r}}=3.76 \mathrm{~min}\right)$; (2) diisopropyl sulfide $\left(t_{\mathrm{r}}=5.55 \mathrm{~min}\right)$; (3) diallyl sulfide ( $t_{\mathrm{r}}=8.37 \mathrm{~min}$ ); (4) di- $t$-butyl sulfide $\left(t_{\mathrm{r}}=9.37 \mathrm{~min}\right)$; (5) di-s-butyl sulfide $\left(t_{\mathrm{r}}=12.35 \mathrm{~min}\right)$; (6) di- $n$-butyl sulfide $\left(t_{\mathrm{r}}=17.52 \mathrm{~min}\right)$; (7) di$n$-hexyl sulfide $\left(t_{\mathrm{r}}=47.12 \mathrm{~min}\right)$.

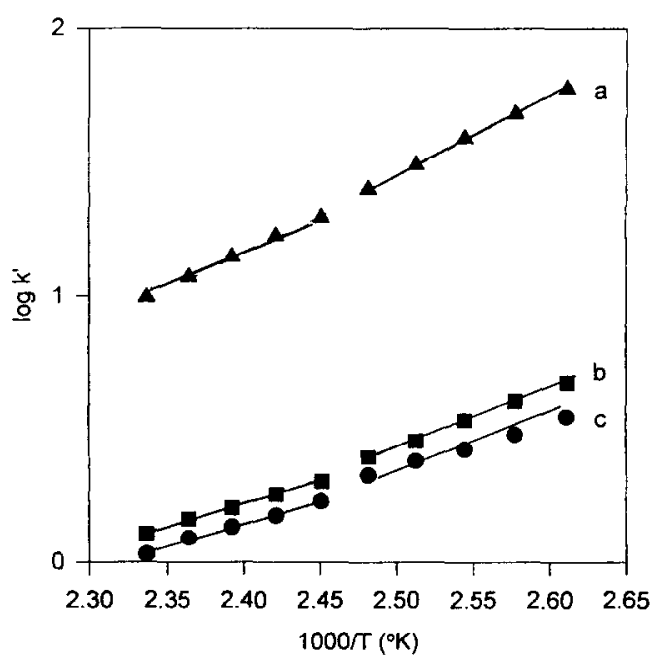

Fig. 5. Van't Hoff plot of dialkyl sulfides. Stationary phase: $5 \%$ DDTBA-Zn coated on Chromosorb; $2.1 \mathrm{~m} \times 3.2 \mathrm{~mm}$ i.d. glass column; Injector temp.: $180^{\circ} \mathrm{C}$; Mobile phase: nitrogen gas, flow rate: $40 \mathrm{ml} \mathrm{min}^{-1}$. Sample: (a) di- $n$-hexyl sulfide; (b) di-n-butyl sulfide; (c) di-t-butyl sulfide. 


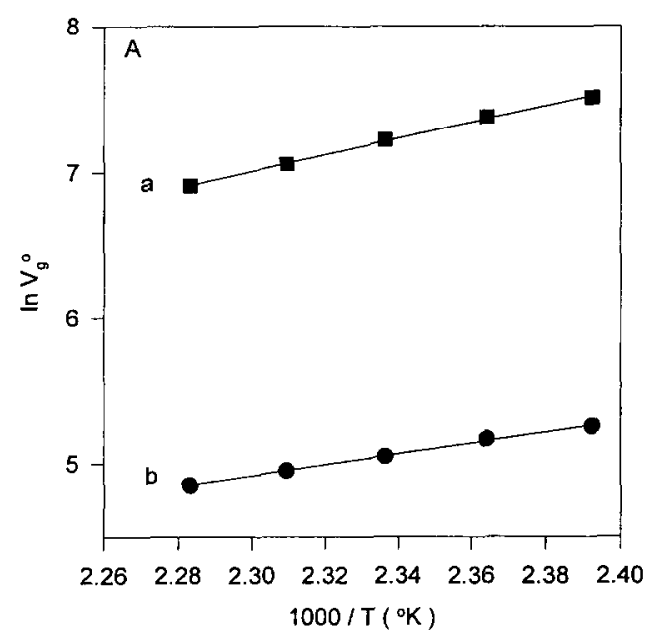

Fig. 6. Temperature dependence of specific retention volume for dialkyl sultides. Conditions as Fig. 5, except stationary phases for (A) is Chromosorb coated with DDTBA-Ni; for (B) is Chromosorb coated with DDTBA-Zn. Sample: (a) di- $n$-hexyl sulfide; (b) di- $n$ butyl sulfide.

the metallomesogens on Chromosorb for dialkyl sulfides.

Fig. 6A and $\mathrm{B}$ illustrate the temperature dependence of the specific retention volumes for di- $n$-hexyl sulfide and di- $n$-butyl sulfide on Chromosorb coated with DDTBA-Ni and DDTBA-Zn, respectively. Enthalpies and entropies for dialkyl sulfides at infinite dilution in the metallomesogens were obtained from the plots. The results are listed in Table 3. In contrast to the PAHs, the sulfur atom in the dialkyl sulfides could form a stronger chemical bond with the central metal atom of the metallomesogens than with the PAHs. Hence both $\Delta H$ and $\Delta S$ are larger than those of the PAHs (Table 3). Comparison of the retention phenomena of dialkyl sulfides (Fig. 4) shows that a longer carbon chain length around the sulfur atom will result in a more dense electron cloud. Similarly, a stronger coordinative bonding between the dialkyl sulfides and the stationary phases will result in a longer retention time. Hence di- $n$-hexyl sulfide has a larger $\Delta \mathrm{H}$ value than di- $n$-butyl sulfide. However, the $\Delta H$ value of di-n-butyl sulfide is slightly larger than that of di-t-butyl sulfide. This might be due to the larger steric hindrance of the $t$ butyl group than the $n$-butyl group.

In the temperature range $140^{\circ}$ to $165^{\circ} \mathrm{C}$, DDTBA$\mathrm{Ni}$ behaves like smectic $\mathrm{H}(\mathrm{SmH})$, and in the
Table 4

Calibration data for polyaromatic hydrocarbons and dialkyl sulfides with conditions as in Table 2

\begin{tabular}{lll}
\hline Compound & $\begin{array}{l}\text { Linear calibration } \\
\text { equation }\end{array}$ & LOD ng $^{-1}$ b \\
\hline $\begin{array}{l}\text { Polyaromatic hydrocarbons } \\
\text { naphthalene }\end{array}$ & $\mathrm{y}=60.56 \mathrm{x}+102.19$ & 8 \\
2-methylnaphthalene & $\mathrm{y}=54.51 \mathrm{x}+100.05$ & 8 \\
1-methylnaphthalene & $\mathrm{y}=54.57 \mathrm{x}+87.59$ & 8 \\
biphenyl & $\mathrm{y}=51.44 \mathrm{x}+114.32$ & 8.5 \\
acenaphthene & $\mathrm{y}=49.27 \mathrm{x}+147.51$ & 8.5 \\
fluorene & $\mathrm{y}=47.30 \mathrm{x}+146.81$ & 9.5 \\
phenanthrene & $\mathrm{y}=34.28 \mathrm{x}+145.90$ & 10.5 \\
anthracene & $\mathrm{y}=34.30 \mathrm{x}+147.40$ & 10.5 \\
Dialkyl sulfides & & \\
diethyl sulfide & $\mathrm{y}=43.49 \mathrm{x}-113.42$ & 10 \\
di-isopropyl sulfide & $\mathrm{y}=40.59 \mathrm{x}-86.19$ & 10 \\
diallyl sulfide & $\mathrm{y}=40.30 \mathrm{x}-146.00$ & 10 \\
di- $t$-butyl sulfide & $\mathrm{y}=37.49 \mathrm{x}-102.85$ & 10 \\
di- $s$-butyl sulfide & $\mathrm{y}=36.55 \mathrm{x}-227.37$ & 11 \\
di- $n$-butyl sulfide & $\mathrm{y}=30.50 \mathrm{x}-121.53$ & 15 \\
\hline
\end{tabular}

${ }^{\mathrm{a}} \mathrm{x}=\mathrm{conc}$ in $\mu \mathrm{g} \mathrm{ml}^{-1}, \mathrm{y}=$ relative peak height, over the range $32-$ $800 \mu \mathrm{g} \mathrm{ml}^{-1} ;{ }^{\mathrm{b}} 3 \sigma$ value.

temperature range of $135^{\circ}$ to $160^{\circ} \mathrm{C}$, DDTBA- $\mathrm{Zn}$ behaves as smectic $\mathrm{C}(\mathrm{SmC})$. Since the structure of $\mathrm{SmH}$ is more compact than that of SmC, the analytes will dissolve more freely in DDTBA-Zn than in DDTBA-Ni.

\subsection{Linear calibration range}

The calibration graphs of peak height against the quantity of each analyte were found to be linear over the concentration range studied, i.e. 32 to $800 \mu \mathrm{g} \mathrm{ml}^{-1}$ for an injection volume of $1 \mu \mathrm{l}$ for all the PAHs and dialkyl sulfides listed in Table 4. The correlation coefficients for all the calibration graphs were between 0.9994 and 0.9998 for the PAHs and between 0.9986 and 0.9993 for the dialkyl sulfides $(n=6)$. The $3 \sigma$ detection limit of most PAHs is less than $10 \mathrm{ng}$, while that of dialkyl sulfides is slightly more than $10 \mathrm{ng}$.

\section{Conclusions}

Separation systems with organic mesogenic stationary phases permit faster and better separations of PAHs, especially in combination with the high 
separation efficiency of capillary columns. However, the work is the first attempt to use DDTBA- $\mathrm{Zn}$ and DDTBA-Ni metallomesogens as stationary phases of ligand exchange gas chromatography for the separations of PAHs and dialkyl sulfides. Both metallomesogens are highly promising for these purpose. From the thermodynamic data, it shows that DDTBA-Zn has a larger affinity with the analytes than DDTBA$\mathrm{Ni}$. The results confirm the assumption that DDTBA$\mathrm{Zn}$ is suitable for the separation of dialkyl sulfides, and DDTBA-Ni for the separation of PAHs. To improve the separation, a ligand exchange mechanism involved both in stationary phase and in mobile phase, or a more efficient capillary column coated with DDTBA-Zn or DDTBA-Ni for the stationary phases have been carried out in our lab [14]. This work will be reported in the subsequent paper (Part 2). As for practical application in environmental sample with the proposed GC system combined with supercritical fluid extraction are now in progress.

\section{Acknowledgements}

We thank the National Science Council of the Republic of China for financial support.

\section{References}

[1] H. Kelker, Fresenius' Z. Anal. Chem., 198 (1963) 254.

[2] G.A. Eiceman, H.H. Hill, Jr. and B. Davani, Anal. Chem., 66 (1994) 621R.

[3] A.M. Giroud and U.T. Mueller-Westerhoff, Mol. Cryst. Liq. Cryst. (Lett.), 41 (1977) 11.

[4] H. Adams, A.C. Albeniz, N.A. Bailey, D.W. Bruce, A.S. Cherodian, R. Dhillon, D.A. Dunmur, P. Espinet, J.L. Feijoo, E. Lalinde, P.M. Maitlis, R.M. Richardson and G. Ungar, J. Mater. Chem., 1 (1991) 843.

[5] C.F. Yeh, S.D. Chyueh, W.S. Chen, J.D. Fang and C.Y. Liu, J. Chromatogr., 630 (1993) 275.

[6] K. Fujimura, M. Kitanaka, H. Takayanagi and T. Ando, Anal. Chem., 54 (1982) 918.

[7] H. Takayanagi, M. Hashizume, K. Fujimura and T. Ando, J. Chromatogr., 350 (1985) 63.

[8] H. Takayanagi, M. Akita, K. Fujimura and T. Ando, J. Chromatogr., 350 (1985) 75.

[9] J. Rayss, Z. Witkiewicz, A. Waksmundzki and R. Babrowski, J. Chromatogr., 188 (1980) 107.

[10] M. Nishioka, B.A. Jones, B.J. Tarbet, J.S. Bradshaw and M.L. Lee, J. Chromatogr., 357 (1986) 79.

[11] D.E. Martire, A. Nikolic and K.L. Vasanth, J. Chromatogr., 178 (1979) 401.

[12] E.F. Meyer, J. Chem. Educ., 50 (1973) 191.

[13] S. Ghodbane, G.A. Oweimreen and D.E. Martire, J. Chromatogr., 556 (1991) 317.

[14] C.C. Hu, The Synthesis and Application of Functional Polymers, Dissertation, National Taiwan University, Taipei, Taiwan, 1995. 